\title{
Size of orthogonal sets of exponentials for the disk
}

\author{
Alex Iosevich and Mihail N. Kolountzakis
}

\begin{abstract}
Suppose that $\Lambda \subseteq \mathbb{R}^{2}$ has the property that any two exponentials with frequency from $\Lambda$ are orthogonal in the space $L^{2}(D)$, where $D \subseteq \mathbb{R}^{2}$ is the unit disk. Such sets $\Lambda$ are known to be finite but it is not known if their size is uniformly bounded. We show that if there are two elements of $\Lambda$ which are distance $t$ apart then the size of $\Lambda$ is $O(t)$. As a consequence we improve a result of Iosevich and Jaming and show that $\Lambda$ has at most $O\left(R^{2 / 3}\right)$ elements in any disk of radius $R$.
\end{abstract}

\section{Introduction}

\subsection{Orthogonal sets of exponentials for domains in Euclidean space}

Let $\Omega \subseteq \mathbb{R}^{d}$ be a bounded measurable set and let us assume for simplicity that $\Omega$ has Lebesgue measure 1. The concept of a spectrum of $\Omega$ that we deal with in this paper was introduced by Fuglede [4], who was studying a problem of Segal on the extendability of the partial differential operators (on $C_{c}^{\infty}(\Omega)$ )

$$
\frac{\partial}{\partial x_{1}}, \frac{\partial}{\partial x_{2}}, \ldots, \frac{\partial}{\partial x_{d}}
$$

to commuting operators on all of $L^{2}(\Omega)$.

Definition 1.1. A set $\Lambda \subseteq \mathbb{R}^{d}$ is called a spectrum of $\Omega$ (and $\Omega$ is said to be a spectral set) if the set of exponentials

$$
E(\Lambda)=\left\{e_{\lambda}(x)=e^{2 \pi i \lambda \cdot x}: \lambda \in \Lambda\right\}
$$

is a complete orthogonal set in $L^{2}(\Omega)$.

(The inner product in $L^{2}(\Omega)$ is $\langle f, g\rangle=\int_{\Omega} f \bar{g}$.)

Mathematics Subject Classification (2010): 42B99.

Keywords: Spectral sets, Fuglede's conjecture. 
It is easy to see (see, for instance, [11]) that the orthogonality of $E(\Lambda)$ is equivalent to the packing condition

$$
\sum_{\lambda \in \Lambda}\left|\widehat{\chi_{\Omega}}\right|^{2}(x-\lambda) \leq|\Omega|^{2}, \quad \text { a.e. }(x),
$$

as well as to the condition

$$
\Lambda-\Lambda \subseteq\{0\} \cup\{\widehat{\chi \Omega}=0\}
$$

Here $\chi_{\Omega}$ is the indicator function of $\Omega$.

The orthogonality and completeness of $E(\Lambda)$ is in turn equivalent to the tiling condition

$$
\sum_{\lambda \in \Lambda}|\widehat{\chi \Omega}|^{2}(x-\lambda)=|\Omega|^{2}, \quad \text { a.e. }(x)
$$

These equivalent conditions follow from the identity

$$
\left\langle e_{\lambda}, e_{\mu}\right\rangle=\int_{\Omega} e_{\lambda} \overline{e_{\mu}}=\widehat{\chi_{\Omega}}(\mu-\lambda),
$$

and from the completeness of all the exponentials in $L^{2}(\Omega)$. Condition (1.1) is roughly expressing the validity of Bessel's inequality for the system of exponentials $E(\Lambda)$, while condition (1.3) says that Bessel's inequality holds as equality.

If $\Lambda$ is a spectrum of $\Omega$ then so is any translate of $\Lambda$, but there may be other spectra as well.

Example: If $Q_{d}=(-1 / 2,1 / 2)^{d}$ is the cube of unit volume in $\mathbb{R}^{d}$ then $\mathbb{Z}^{d}$ is a spectrum of $Q_{d}$. Let us remark here that there are spectra of $Q_{d}$ which are very different from affine images of the lattice $\mathbb{Z}^{d}$ ([8], [16], [10]).

Research on spectral sets [17], [15], [14] has been driven for many years by a conjecture of Fuglede [4], sometimes called the Spectral Set Conjecture, which stated that a set $\Omega$ is spectral if and only if it is a translational tile. A set $\Omega$ is a translational tile if we can translate copies of $\Omega$ around and fill space without overlaps. More precisely, there exists a set $S \subseteq \mathbb{R}^{d}$ such that

$$
\sum_{s \in S} \chi_{\Omega}(x-s)=1, \quad \text { a.e. }(x) .
$$

One can generalize naturally the notion of translational tiling from sets to functions by saying that a nonnegative $f \in L^{1}\left(\mathbb{R}^{d}\right)$ tiles when translated at the locations $S$ if $\sum_{s \in S} f(x-s)=\ell$ for almost every $x \in \mathbb{R}^{d}$ (the constant $\ell$ is called the level of the tiling). Thus the question of spectrality for a set $\Omega$ is essentially a tiling question for the function $\left|\widehat{\chi_{\Omega}}\right|^{2}$ (the power-spectrum). Taking into account the equivalent condition (1.3) one can now, more elegantly, restate the Fuglede Conjecture as the equivalence

$$
\chi_{\Omega} \text { tiles } \mathbb{R}^{d} \text { by translation at level } 1
$$

$$
\Longleftrightarrow\left|\widehat{\chi_{\Omega}}\right|^{2} \text { tiles } \mathbb{R}^{d} \text { by translation at level }|\Omega|^{2} \text {. }
$$


In this form the conjectured equivalence is perhaps more justified. However this conjecture is now known to be false in both directions if $d \geq 3$ (see [19], [18], [12], [13], [2] and [3]), but remains open in dimensions 1 and 2, and it is not out of the question that the conjecture is true in all dimensions if one restricts the domain $\Omega$ to be convex. (It is known that the direction "tiling $\Rightarrow$ spectrality" is true in the case of convex domains; see for instance [11].) The equivalence (1.5) is also known, from the time of Fuglede's paper [4], to be true if one adds the word lattice to both sides (that is, lattice tiles are the same as sets with a lattice spectrum).

\subsection{Orthogonal exponentials for the disk}

Already in [4] it was claimed that the disk in the plane (and the Euclidean ball in $\mathbb{R}^{d}$ ) is not a spectral set, in agreement with (1.5). A proof appeared in [7]. Later it was proved in [5] and [9] that any orthogonal set of exponentials for the ball must necessarily be finite. It is still unknown however if there is a uniform bound for the size of each orthogonal set. It is still a possibility that there are arbitrarily large orthogonal sets of exponentials for the ball, and proving a uniform upper bound is probably very hard as it appears to depend on algebraic relations among the roots of the Bessel function $J_{1}$. In the direction of showing upper bounds for orthogonal sets of exponentials, it was proved in [6] that if $\Lambda$ is a set of orthogonal exponentials for the ball then $\left|\Lambda \cap[-R, R]^{d}\right|=O(R)$, with the implicit constant independent of $\Lambda$. Completeness would of course require that $\left|\Lambda \cap[-R, R]^{d}\right| \gtrsim R^{d}$ (this follows easily from the tiling condition (1.3)).

The result in this paper, Theorem 1.2 below, improves the result of [6] mentioned above. We choose to work only in the case of the unit disk in the plane and not in higher dimension or in the larger class of smooth convex bodies in order to present a clear geometric argument, which probably extends to these cases as well.

Abusing language slightly, for the benefit of readability, let us also call two vectors $\lambda$ and $\mu$ orthogonal if the corresponding exponentials $e_{\lambda}(x)$ and $e_{\mu}(x)$ are orthogonal in $L^{2}(\Omega)$. In other words, we will often identify the frequency $\lambda$ with the exponential $e_{\lambda}(x)$.

Theorem 1.2. There are constants $C_{1}, C_{2}$ such that whenever $\Lambda \subseteq \mathbb{R}^{2}$ is an orthogonal set of exponentials for the unit disk in the plane and

$$
t=\inf \{|\lambda-\mu|: \lambda, \mu \in \Lambda, \lambda \neq \mu\}
$$

then

$$
|\Lambda| \leq C_{1} t
$$

(It is well known and easy to see from (1.1) or (1.2) that $t>0$.)

Furthermore,

$$
\left|\Lambda \cap[-R, R]^{2}\right| \leq C_{2} R^{2 / 3} \quad \text { for all } R \geq 1
$$

The proof of Theorem 1.2 is given in the next section. 


\section{Proof of the main theorem}

A crucial ingredient of the proof is the asymptotics for the zeros of the Fourier transform of the indicator function of the unit disk $D=\left\{x \in \mathbb{R}^{2}:|x| \leq 1\right\}$, which is of course a radial function. Since the zeros of $\widehat{\chi_{D}}(r)$ are the same as the zeros of the Bessel function $J_{1}(2 \pi r)$, and since for the zeros of $J_{1}$, written as $j_{1, n}$, $n=1,2, \ldots$, we have ([1]) an asymptotic expansion

$$
j_{1, n}=\rho_{n}+\frac{K_{1}}{\rho_{n}}+O\left(\frac{1}{\rho_{n}^{3}}\right), \quad \text { where } \rho_{n}=n \pi+\frac{\pi}{4}, n=1,2, \ldots,
$$

where $K_{1}$ is an absolute constant, it follows that the zeros of $\widehat{\chi_{D}}(r)$ are at the locations

$$
r_{n}=\frac{1}{2 \pi} j_{1, n}=\frac{n}{2}+\frac{1}{8}+\frac{K_{1}}{2 \pi \rho_{n}}+O\left(n^{-3}\right) .
$$

Moreover, if $0 \leq m-n \leq K$ and $m, n \rightarrow \infty$, it follows from (2.1) that

$$
\begin{aligned}
r_{m}-r_{n} & =\frac{m-n}{2}+O\left(K n^{-2}\right)=\frac{m-n}{2}+O\left(K r_{n}^{-2}\right) \\
& =\frac{m-n}{2}+O\left(\left(r_{m}-r_{n}\right) r_{n}^{-2}\right) .
\end{aligned}
$$

Lemma 2.1. There are constants $R_{0}, C>0$ such that whenever $a, b, c \in \mathbb{R}^{2}$ are orthogonal for the unit disk, with $|a-c|,|b-c|,|a-b| \geq R \geq R_{0}$, then the two largest angles of the triangle abc (as well as all its external angles) are

$$
\geq \frac{C}{R^{1 / 2}}
$$

Proof. Assume without loss of generality that $R=|a-c| \leq|b-c| \leq|a-b|$ (see Figure 1).

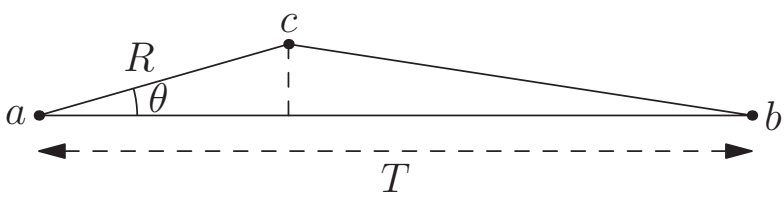

FiguRE 1. Three points orthogonal for the unit disk.

Writing $\theta=\widehat{b a c}$ for the second largest angle and $T=|a-b|$ we have

$$
|b-c|=\sqrt{(T-R \cos \theta)^{2}+R^{2} \sin ^{2} \theta}=\sqrt{(T-R)^{2}+2 T R(1-\cos \theta)},
$$

from which we get

$$
|b-c|-(T-R)=\frac{2 T R(1-\cos \theta)}{T-R+|b-c|}=\frac{2 R(1-\cos \theta)}{1-\frac{R}{T}+\frac{|b-c|}{T}} \leq 2 R(1-\cos \theta) \leq R \theta^{2}
$$


From (2.2) it follows that as $R \rightarrow \infty$ the quantities $|a-b|,|b-c|,|a-c|$ are all of the form

$$
\frac{k}{2}+\frac{1}{8}+o(1), \quad \text { for some integer } k \text {. }
$$

It follows that $|b-c|-(T-R)=k / 2+1 / 8+o(1)$, for some integer $k \geq 0$. This, together with (2.5), implies that $k / 2+1 / 8+o(1) \leq R \theta^{2}$, which gives us the required inequality with constant $C$ arbitrarily close to $\sqrt{1 / 8}$ when $R$ is large.

Remark 2.2. It follows from (1.2) that whenever $\Lambda$ is an orthogonal set for the disk the distance of any two points of $\Lambda$ is bounded below by a constant $c_{0}$, independent of $\Lambda$. It is not hard to see that any orthogonal set may be partitioned into a constant number of subsets $\Lambda_{j}$ such that the distance of any two points of $\Lambda_{j}$ is bounded below by $R_{0}$, the constant mentioned in Lemma 2.1. It is also clear that it suffices to prove Theorem 1.2 for orthogonal sets that are $R_{0}$-separated. The validity of the theorem in the general case follows easily if one only alters the constants.

Assumption: For this reason we will assume from now on that we are dealing with a $R_{0}$-separated orthogonal set, even when we do not say so explicitly.

Corollary 2.3. There is a constant $C^{\prime}>0$ such that whenever $a, b, c \in \mathbb{R}^{2}$ belong to a $R_{0}$-separated orthogonal set for the unit disk and their pairwise distances are at least $L$, then they cannot all belong to a strip of width $C^{\prime} L^{1 / 2}$.

Proof. Suppose they do belong to such a strip.

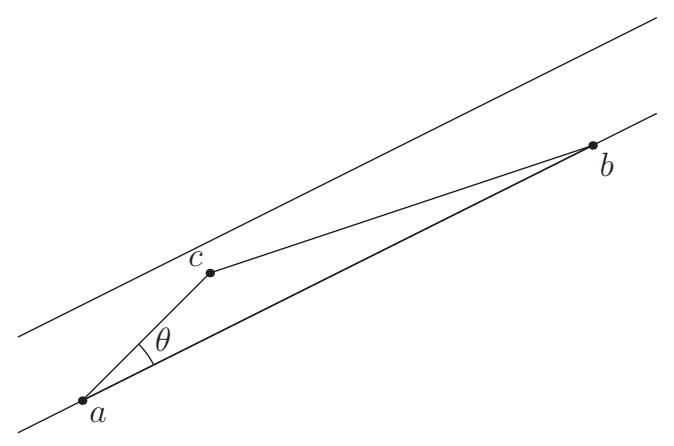

FiguRE 2. Three points in a strip.

Move and turn the strip so that two of the points, those with the largest distance apart, say $a$ and $b$, are on one of the strip sides, and the other point $c$ is still in the strip (see Figure 2). Assume also that $c$ is closer to $a$ than to $b$. By Lemma 2.1 it follows that the angle $\theta=\widehat{b a c}$ is at least

$$
\frac{C}{|a-c|^{1 / 2}},
$$

from which we obtain that the distance of $c$ to the line $a b$ is at least $C \sqrt{a-c} \geq$ $C \sqrt{L}$, a contradiction if the constant $C^{\prime}$ in Corollary 2.3 is sufficiently small. 
Corollary 2.4. Suppose $\Lambda \subseteq \mathbb{R}^{2}$ is a $R_{0}$-separated set of orthogonal exponentials for the unit disk, $R>0$ and let

$$
\Delta=\inf \left\{|\lambda-\mu|: \lambda, \mu \in \Lambda \cap[-R, R]^{2}\right\} .
$$

Then, for some constant $C>0$,

$$
\left|\Lambda \cap[-R, R]^{2}\right| \leq C \frac{R}{\Delta^{1 / 2}},
$$

Proof. Cover $[-R, R]^{2}$ by $O\left(R / \Delta^{1 / 2}\right)$ strips of width $c \Delta^{1 / 2}$, for small $c>0$. From Corollary 2.3, each of these contains at most two points of $\Lambda$.

We may assume from now on that the points $V=(\Delta, 0)$ and $-V=(-\Delta, 0)$ belong to the set $\Lambda$ and that $t / 2 \leq \Delta \leq t$. It is also sufficient to bound the size of $\Lambda$ in the first quadrant only, for reasons of symmetry, so we restrict ourselves to the first quadrant. By Corollary 2.3 we have that

$$
|\Lambda \cap\{(x, y): x, y \geq 0, \min \{x, y\} \leq \Delta\}|=O\left(\Delta^{1 / 2}\right) .
$$

So from now on we may assume that the point $\lambda=(x, y) \in \Lambda$ belongs to the first quadrant, and has $x, y \geq \Delta$.

To each $\lambda=(x, y)$ in the open first quadrant we correspond two numbers $a(\lambda), b(\lambda) \in(0, \Delta)$ such that $a(\lambda)^{2}+b(\lambda)^{2}=\Delta^{2}$ and $\lambda$ is on the hyperbola

$$
\mathfrak{H}_{\lambda}: \frac{x^{2}}{a(\lambda)^{2}}-\frac{y^{2}}{b(\lambda)^{2}}=1, \quad(x, y \geq 0) .
$$

This hyperbola $\mathfrak{H}_{\lambda}$ is the locus of all points $p$ in the first quadrant such that

$$
|p+V|-|p-V|=2 a(\lambda) .
$$

A parametrization of $\mathfrak{H}_{\lambda}$ is

$$
x(s)=a(\lambda) \cosh s, \quad y(s)=b(\lambda) \sinh s, \quad(s \geq 0) .
$$

It follows that in the region of interest $x, y \geq \Delta$ we have

$$
\Delta \leq x \leq a(\lambda) e^{s} \leq 2 x \leq 2|\lambda|, \quad \Delta \leq y \leq b(\lambda) \frac{e^{s}}{2} \leq 2 y \leq 2|\lambda| .
$$

Lemma 2.5. There is a constant $K>0$ such that $b(\lambda) \geq K \Delta^{1 / 2}$ with the exception of at most a constant number of points of $\Lambda$.

Proof. It follows from Lemma 2.1 that $\lambda$ cannot belong to the sector defined by the $x$-axis from $V$ onward and the straight line $M$ through $V$ of angle $c \Delta^{-1 / 2}$, if $c>0$ is small enough (refer to Figure 3 ). Now draw a parallel line $L$ to straight line $M$ through the origin and note that the strip bordered by these two parallel lines, $L$ and $M$, has width $O\left(\Delta^{1 / 2}\right)$. Therefore, by Corollary 2.3 , there is only a constant number of elements of $\Lambda$ that can belong to the sector defined by the positive $x$-semiaxis and the straight line $L$ (shaded region in Figure 3 ). 


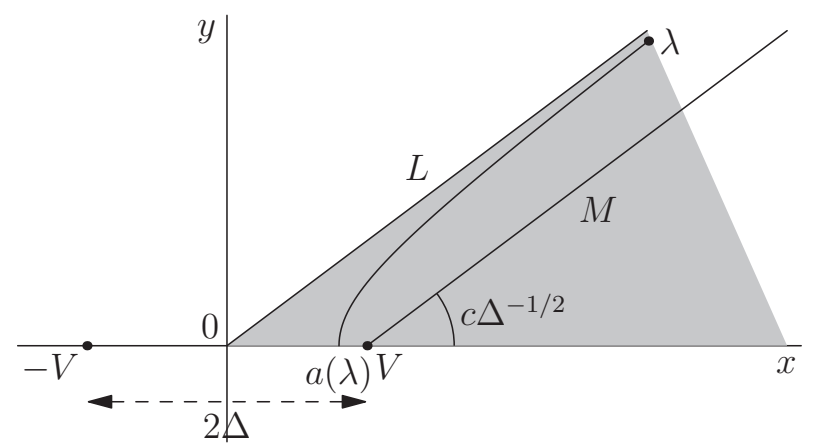

Figure 3. Figure to aid the proof of Lemma 2.5.

Suppose now that $\lambda \in \Lambda$ is such that $b(\lambda) \leq K \Delta^{1 / 2}$, for an appropriately small constant $K$, so that we also have $a(\lambda) \geq \Delta / 2$. It follows that the asymptote to the hyperbola $\mathfrak{H}_{\lambda}$, with equation $y=(b(\lambda) / a(\lambda)) x$, has slope at most $2 K \Delta^{-1 / 2}$, which implies that $\lambda$, lying below that asymptote, is contained in the (shaded) sector mentioned above.

Writing $H(a, \Delta)$ for the hyperbola $x^{2} / a^{2}-y^{2} / b^{2}=1$, with $a^{2}+b^{2}=\Delta^{2}$, we consider the finite family of confocal hyperbolas (we consider the special case $k=0$ as a hyperbola too; it is the perpendicular bisector of the line segment connecting the foci)

$$
H_{k}=H\left(\frac{k}{4}, \Delta\right), \quad k=0,1,2, \ldots,\lfloor 4 \Delta\rfloor .
$$

The hyperbola $H_{k}$ is the locus of all points $p$ with $|p+V|-|p-V|=k / 2$. For each $\lambda$ we define the corresponding $k$ to be the unique integer such that

$$
|\lambda+V|-|\lambda-V|=\frac{k}{2}+2 \varepsilon, \quad-\frac{1}{8} \leq \varepsilon<\frac{1}{8} .
$$

We write $a=k / 4, b=\sqrt{\Delta^{2}-a^{2}}$. It follows from (2.8) and (2.12) that

$$
a(\lambda)=a+\varepsilon, \quad b(\lambda)=b-\varepsilon^{\prime},
$$

for some $\varepsilon^{\prime}$, of the same sign as $\varepsilon$. From (2.3) we have that

$$
|\varepsilon| \leq C \Delta|\lambda|^{-2},
$$

for some absolute finite constant $C>0$.

Next we estimate $\varepsilon^{\prime}$ :

$$
\begin{aligned}
\left|\varepsilon^{\prime}\right| & =|b-b(\lambda)|=\left|\sqrt{\Delta^{2}-a^{2}}-\sqrt{\Delta^{2}-a(\lambda)^{2}}\right| \\
& =\left|\frac{a(\lambda)^{2}-a^{2}}{b+b(\lambda)}\right|=\left|\varepsilon \frac{a+a(\lambda)}{b+b(\lambda)}\right| \leq|\varepsilon| \frac{2 \Delta}{b(\lambda)} \\
& =O\left(|\varepsilon| \Delta^{1 / 2}\right) \quad\left(\text { from Lemma } 2.5, \text { excepting finitely many } \lambda^{\prime} \text { 's }\right) \\
& =O\left(\Delta^{3 / 2}|\lambda|^{-2}\right) \quad(\text { from }(2.13)) .
\end{aligned}
$$


The asymptote $L(a, \Delta)$ to $H(a, \Delta)=H_{k}$ is the line $y=(b / a) x$ and a unit normal vector to this line is $u=(b / \Delta,-a / \Delta)$. We can bound the distance of

$$
\lambda=(x, y)=(a(\lambda) \cosh s, b(\lambda) \sinh s)
$$

to $L(a, \Delta)$ as follows:

$$
\begin{aligned}
|u \cdot \lambda| & =\left|\frac{b x}{\Delta}-\frac{a y}{\Delta}\right|=\frac{1}{\Delta}|b a(\lambda) \cosh s-a b(\lambda) \sinh s| \\
& =\frac{1}{\Delta}\left|\left(b(\lambda)+\varepsilon^{\prime}\right) a(\lambda) \cosh s-(a(\lambda)-\varepsilon) b(\lambda) \sinh s\right| \\
& =\frac{1}{\Delta}\left|a(\lambda) b(\lambda) e^{-s}+\varepsilon b(\lambda) \sinh s+\varepsilon^{\prime} a(\lambda) \cosh s\right| \\
& =O\left(\Delta^{2}|\lambda|^{-1}\right)+|\varepsilon| O\left(\Delta^{-1} b(\lambda) \sinh s+\Delta^{-1 / 2} a(\lambda) \cosh s\right) \\
& \quad\left(\text { since } \varepsilon^{\prime}=O\left(\Delta^{1 / 2} \varepsilon\right) \text { and } a(\lambda) e^{s} \sim|\lambda| \text { or } b(\lambda) e^{s} \sim|\lambda| \text { from }(2.10)\right) \\
& =O\left(\Delta^{2}|\lambda|^{-1}\right)+|\varepsilon| O\left(\Delta^{-1} b(\lambda) e^{s}+\Delta^{-1 / 2} a(\lambda) e^{s}\right) \\
& =O\left(\Delta^{2}|\lambda|^{-1}\right)+O\left(|\varepsilon||\lambda| \Delta^{-1 / 2}\right) \quad(\text { from }(2.13)) .
\end{aligned}
$$

Therefore in the region $|\lambda| \geq C \Delta^{3 / 2}$ each point of $\Lambda$ is at distance $O\left(\Delta^{1 / 2}\right)$ from one of the asymptotes to the hyperbolas $H_{k}$. In each strip of width $O\left(\Delta^{1 / 2}\right)$ around each such asymptote we therefore have at most $C$ points, a constant. This gives a total of $O(\Delta)$ points of $\Lambda$ in that region as there are that many hyperbolas $H_{k}$. In the region $|\lambda| \leq C \Delta^{3 / 2}$ we also have $O(\Delta)$ points because of Corollary 2.4. This concludes the proof of the first part of Theorem 1.2.

To prove that $\left|\Lambda \cap[-R, R]^{2}\right|=O\left(R^{2 / 3}\right)$ notice that by Corollary 2.4 and by the first part of Theorem 1.2 we have

$$
\left|\Lambda \cap[-R, R]^{2}\right|=O\left(\min \left\{\frac{R}{t^{1 / 2}}, t\right\}\right)=O\left(R^{2 / 3}\right) .
$$

\section{References}

[1] Abramowitz, M. And Stegun, I. A.: Handbook of mathematical functions with formulas, graphs and mathematical tables. National Bureau of Standards Applied Mathematics Series 55, US Government Printing Office, Washington, DC, 1964.

[2] Farkas, B., Matolcsi, M. and Móra, P.: On Fuglede's conjecture and the existence of universal spectra. J. Fourier Anal. Appl. 12 (2006), no. 5, 483-494.

[3] Farkas, B. And RÉvész, S.: Tiles with no spectra in dimension 4. Math. Scand. 98 (2006), no. 1, 44-52.

[4] Fuglede, B.: Commuting self-adjoint partial differential operators and a group theoretic problem. J. Funct. Anal. 16 (1974), 101-121.

[5] Fuglede, B.: Orthogonal exponentials on the ball. Exposition. Math. 19 (2001), no. $3,267-272$. 
[6] Iosevich, A. And Jaming, P.: Distance sets that are a shift of the integers and Fourier basis for planar convex sets. Acta Math. Hungar. 121 (2008), no. 1-2, $107-118$.

[7] Iosevich, A., Katz, N. And Pedersen, S.: Fourier bases and a distance problem of Erdös. Math. Res. Lett. 6 (1999), no. 2, 251-255.

[8] Iosevich, A. And Pedersen, S.: Spectral and tiling properties of the unit cube. Internat. Math. Res. Notices 1998 (1998), no. 16, 819-828.

[9] Iosevich, A. And Rudnev, M.: A combinatorial approach to orthogonal exponentials. Internat. Math. Res. Notices 2003 (2003), no. 50, 2671-2685.

[10] Kolountzakis, M.: Packing, tiling, orthogonality and completeness. Bull. London Math. Soc. 32 (2000), no. 5, 589-599.

[11] Kolountzakis, M.: The study of translational tiling with Fourier Analysis. In Fourier analysis and convexity, 131-187. Birkhauser, 2004.

[12] Kolountzakis, M. And Matolcsi, M.: Complex Hadamard matrices and the spectral set conjecture. Collect. Math., vol. extra (2006), 281-291.

[13] Kolountzakis, M. And Matolcsi, M.: Tiles with no spectra. Forum Math. 18 (2006), 519-528.

[14] ŁabA, I.: Fuglede's conjecture for a union of two intervals. Proc. Amer. Math. Soc. 129 (2001), 2965-2972.

[15] ŁabA, I.: The spectral set conjecture and multiplicative properties of roots of polynomials. J. London Math. Soc. 65 (2002), 661-671.

[16] Lagarias, J., Reeds, J. And Wang, Y.: Orthonormal bases of exponentials for the $n$-cube. Duke Math. J. 103 (2000), no. 1, 25-38.

[17] Lagarias, J. And Wang, Y.: Spectral sets and factorizations of finite abelian groups 1. J. Funct. Anal. 145 (1997), no. 1, 73-98.

[18] Matolcsi, M.: Fuglede's conjecture fails in dimension 4. Proc. Amer. Math. Soc. 133 (2005), no. 10, 3021-3026.

[19] TAO, T.: Fuglede's conjecture is false in 5 and higher dimensions. Math. Res. Lett. 11 (2004), no. 2-3, 251-258.

Received November 7, 2011; revised March 24, 2012.

Alex Iosevich: Department of Mathematics, 915 Hylan Building, University of Rochester, Rochester, NY 14627, USA.

E-mail: iosevich@math.rochester.edu

Minail N. Kolountzakis: Department of Mathematics, University of Crete, 71003 Iraklio, Crete, Greece.

E-mail: kolount@math.uoc.gr

A. I. supported by NSF grant DMS10-45404. M. K. supported by research grant no. 3223 from the University of Crete and by NSF grant DMS10-45404 and grants of the University of Rochester, whose hospitality is gratefully acknowledged. 